\title{
TRANSPLANTE HEPÁTICO ORTOTÓPICO EXPERIMENTAL EM SUÍNOS: RESULTADOS INICIAIS (I)
}

\author{
Experimental orthotopic liver transplantation in swine: initials results
}

\begin{abstract}
Orlando Jorge Martins Torres ${ }^{1}$, Érica Sampaio Barbosa ${ }^{2}$, Noelia Dias Carneiro Barros ${ }^{2}$, Patrícia Brandão Pantoja ${ }^{2}$, Elisabeth Teixeira Noguera Servin ${ }^{3,4}$, Poliana Cristina Oliveira Moreira ${ }^{2}$, Edson Dener Zandonadi Ferreira ${ }^{2}$, Raquel Aranha Viegas ${ }^{2}$, Cristiany de Almeida Barros ${ }^{2}$, Santiago Cirilo Noguera Servin ${ }^{3}$, José Aparecido Valadão ${ }^{3}$, Tereza Rachel Vieira Gomes ${ }^{3}$, Natalino Salgado Filho $^{3}$, Waston Gonçalves Ribeiro ${ }^{3,4}$
\end{abstract}

\section{RESUMO}

Objetivo: Este estudo tem como objetivo apresentar os resultados iniciais do transplante hepático ortotópico experimental em suínos da Universidade Federal do Maranhão. Material e Métodos: No período de abril de 2004 a abril 2005 foram realizados 18 transplantes de fígado utilizando 36 porcos fêmeas, raça Landrace, pesando entre 32 e $38 \mathrm{Kg}$. Estes animais foram divididos em dois grupos, doador e receptor, que receberam enxerto total. O procedimento cirúrgico foi dividido em quatro fases: captação, operação de mesa (back-table), hepatectomia do receptor e reimplantação do fígado. Os dados analisados foram a pressão arterial média, pressão venosa central, freqüência cardíaca, $\mathrm{pO}_{2}, \mathrm{pCO}_{2}, \mathrm{HCO}_{3}$, excesso de base, $\mathrm{SO}_{2}, \mathrm{pH}, \mathrm{Na}+, \mathrm{K}+, \mathrm{Cl}-$ e $\mathrm{Ca}++$. Registramos o tempo da cirurgia, obtenção da perfusão hepática, isquemia fria, back table, fase anepática e o tempo para realização das anastomoses. Resultados: Observamos um tempo longo de isquemia fria do enxerto, entretanto as anastomoses da veia cava supra-hepática e veia porta foram consideradas satisfatórias resultando em uma fase anepática curta. Foram observadas alterações clínicas e gasométricas importantes na fase de reperfusão do enxerto. Valores laboratoriais do sódio e cloro séricos estiveram alterados nas fases anepática e de reperfusão do enxerto. Conclusão: $O$ transplante hepático ortotópico experimental em suínos é capaz de otimizar a técnica cirúrgica bem com estimular o desenvolvimento de pesquisas em transplante hepático.

Descritores: transplante hepático, cirurgia experimental, cirurgia hepática, suínos.

Trabalho realizado no Programa de Transplante Hepático Experimental do Hospital Universitário da Universidade Federal do Maranhão - UFMA. Patrocinado pelo CNPq - Conselho Nacional de Desenvolvimento Científico e Tecnológico.

1. Professor Livre-Docente do Departamento de Cirurgia da Universidade Federal do Maranhão - UFMA

2. Estudantes de Medicina - UFMA, Bolsistas do PIBIC-CNPq-UFMA

3. Médicos do Programa de Transplante Hepático Experimental - HUPD-UFMA

4. Mestrando em Ciências da Saúde - UFMA

Endereço para correspondência: Dr. Orlando Torres - Rua dos Bicudos 14/600 Ed. Aspen - Renascença II - CEP: 65075-090 - São Luís - MA - Brasil - Fone (98) 3227-4009 Fax (98) 3227-4073 - E-mail: o.torres@uol.com.br

Recebido em: 20/06/05

Aceito em: 05/07/05

\section{INTRODUÇÃO}

O transplante hepático tem sido largamente utilizado e é o tratamento de escolha para uma variedade de doenças crônicas do fígado, em estágio terminal, bem como para doença hepática severa aguda para as quais nenhuma outra terapia satisfatória pode ser disponível. Devido à melhora da técnica cirúrgica e da utilização de novos agentes imunossupressores, este tratamento tem sido bem aceito e, conseqüentemente, o número de pacientes em lista de espera, bem como tempo de espera em lista para transplante de fígado, tem aumentado. ${ }^{1,2,3,4}$

Desde o primeiro transplante hepático clínico realizado em 1963, uma grande revolução tem sido observada, com os resultados cada vez mais promissores. Este procedimento terapêutico também tem sido utilizado de forma eficiente no Brasil, com evolução satisfatória desde o seu início em $1968 .{ }^{5,6} \mathrm{O}$ interesse atual no transplante hepático como forma de tratamento destes pacientes tem estimulado a implantação de novos programas de transplante clínico. Devido à complexidade técnica envolvida no transplante hepático, o treinamento das equipes anestésico-cirúrgicas através de cirurgias experimentais em animais tem sido considerado como passo fundamental antes de sua aplicação clínica. ${ }^{7,8,9,10,11}$ Ao mesmo tempo, o desenvolvimento da pesquisa em transplante hepático depende deste procedimento em animais. Desta forma, os propósitos de um programa de transplante hepático experimental tem sido o de treinar uma equipe especializada nos 
306 Orlando Jorge Martins Torres, Érica Sampaio Barbosa, Noelia Dias Carneiro Barros, Patrícia Brandão Pantoja, Elisabeth Teixeira Noguera Servin, Poliana Cristina Oliveira Moreira, Edson Dener Zandonadi Ferreira, Raquel Aranha Viegas, Cristiany de Almeida Barros, Santiago Cirilo Noguera Servin, José Aparecido Valadão, Tereza Rachel Vieira Gomes, Natalino Salgado Filho, Waston Gonçalves Ribeiro

aspectos técnicos que este procedimento tanto necessita e para realização de pesquisas experimentais ligadas ao transplante $12,13,14,15,16,17$. Este estudo tem como objetivo apresentar os resultados iniciais do programa de transplante hepático ortotópico experimental em suínos da Universidade Federal do Maranhão.

\section{MATERIAL E MÉTODO}

Este estudo foi aprovado pelo comitê de ética e pesquisa experimental em animais da Universidade Estadual do Maranhão (UEMA). Os transplantes foram realizados no Laboratório de Pesquisas em Cirurgia Experimental do Hospital Universitário da Universidade Federal do Maranhão (UFMA). Foram obedecidos os princípios internacionais de pesquisa em animais, do Colégio Brasileiro de Experimentação Animal, sendo as cirurgias supervisionadas por um médico veterinário.

No período de 18 de abril de 2004 a 24 de abril 2005 foram realizados 18 transplantes de fígado, utilizando 36 porcos fêmeas, raça Landrace, pesando entre 32 e $38 \mathrm{Kg}$. Estes animais foram divididos em dois grupos, doador e receptor, estes últimos recebendo enxerto total. Os animais foram mantidos sob condições laboratoriais padrão e em jejum por 24 horas antes da cirurgia.Todos os animais foram submetidos ao transplante hepático sob anestesia geral. 18,19 Foi utilizada a técnica clássica, não sendo, portanto, realizado o Piggy-Back. O procedimento cirúrgico foi dividido em quatro fases: captação (operação do doador), cirurgia de mesa (back-table), hepatectomia do receptor e reimplantação do fígado.

\section{OPERAÇÃO DO DOADOR}

Realizada incisão mediana, dissecção e cateterização da aorta abdominal acima da bifurcação das artérias ilíacas. A aorta ao nível do diafragma é clampeada e iniciado o resfriamento com um litro de solução de Ringer Lactato a $4^{\circ} \mathrm{C}$ pela aorta e um litro de solução de preservação (EuroCollins) a $4^{\circ} \mathrm{C}$ pela veia esplênica. A vesícula biliar era aberta enxaguada com solução de Ringer Lactato a $4^{\circ} \mathrm{C}$

A dissecção do ligamento hepatoduodenal continua com o preparo da veia porta abaixo de sua junção com a veia esplênica. A aorta é seccionada acima e abaixo do tronco celíaco para retirada da artéria hepática com o patch de Carrel. A veia cava foi removida junto com $10 \mathrm{~mm}$ de tecido diafragmático. $\mathrm{O}$ fígado retirado foi colocado em saco plástico com solução de Eurocollins a $4^{\circ} \mathrm{C}$ e colocado em uma bacia para o back-table.

\section{BACK-TABLE}

A perfusão com solução de Euro-Collins a $4^{\circ} \mathrm{C}$ é realizado através da veia porta e artéria celíaca para conservação. Realizado chuleios semicirculares do manguito diafragmático da veia cava suprahepática. Todas as tributárias dos grandes vasos são ligadas e a permeabilidade controlada.

\section{HEPATECTOMIA DO RECEPTOR}

A artéria carótida comum direita é cateterizada para mensuração da pressão arterial média e coleta de sangue arterial sistêmico, a veia jugular interna esquerda é cateterizada para administração de líquidos e para o by-pass veno-venoso durante a fase anepática e a veia jugular externa direita é cateterizada para administração de líquidos, medicamentos, sangue (coletado do animal doador) e monitoramento da pressão venosa central (PVC).
Realiza-se a dissecção e cateterização da veia ilíaca comum esquerda e veia esplênica para confecção do by-pass veno-venoso. Inicia-se a dissecção do ligamento hepatoduodenal, o ducto hepático comum é dividido próximo ao fígado, após ligadura distal. A artéria hepática comum é dissecada e realizada ligadura alta. A veia porta é dissecada na junção com a veia esplênica. Em seguida, era procedida a liberação da veia cava infra-hepática. A veia porta e a veia cava inferior infrahepática são clampeadas. Neste momento o by-pass passivo venovenoso em Y da veia ilíaca esquerda e veia esplênica em direção à veia jugular interna esquerda está completamente funcionante. $O$ fígado do receptor é removido.

\section{IMPLANTE DO ENXERTO}

O fígado, previamente perfundido com dois litros de solução de Ringer Lactato através da veia porta, é posicionado e a anastomose da veia cava supra-hepática é realizada com sutura contínua posterior com fio de Polipropileno 4.0, com duas agulhas. A sutura anterior inicia-se também à esquerda. Em seguida, a anastomose da veia porta é realizada da mesma forma com Polipropileno 5.0, de duas agulhas. Os nós com fator de crescimento no ângulo direito da veia porta terminam o procedimento. Os clamps da veia porta são liberados, caracterizando o fim da fase anepática. A anastomose da veia cava infra-hepática é realizada seguindo o mesmo procedimento e retira-se os sistema de by-pass veno-venoso. Realiza-se a anastomose terminoterminal da artéria hepática com pontos separados de Polipropileno 7.0 e anastomose bilio-digestiva do tipo colecisto-duodenostomia com sutura contínua com fio de Poliglactina 910, 3.0.

\section{MONITORIZAÇÃO}

A monitoração dos animais é realizada com cardioscópio com cinco derivações, oximetria, e cardioscopia. Amostra de sangue arterial do animal receptor é coletado no momento da canulação da artéria (primeira amostra), no início da fase anepática (segunda amostra) e logo após a reperfusão do fígado (terceira amostra). Os dados analisados a partir destes parâmetros foram a pressão arterial média (PAM), pressão venosa central (PVC), freqüência cardíaca, pO2, $\mathrm{pCO}_{2}, \mathrm{HCO}_{3}$, excesso de base (BE), $\mathrm{SO}_{2}, \mathrm{Na}+, \mathrm{K}+, \mathrm{Cl}$ - e $\mathrm{Ca}++$. Registramos o tempo gasto em cada etapa do procedimento no animal doador e receptor como cirurgia, obtenção da perfusão hepática, isquemia fria, back table, fase anepática bem como o tempo para realização das anastomoses.

Foram excluídos do estudo os dois primeiros transplantes que fizeram parte do projeto piloto, sendo considerados então, 16 transplantes com 32 animais.

\section{RESULTADOS}

A cirurgia do doador para retirada do fígado para transplante foi realizada de forma satisfatória em um tempo considerado ideal, entretanto foi observado um tempo de isquemia fria do órgão relativamente longo (tabela 1 ).

Tabela 1. Tempo médio (em minutos) gasto para realização das etapas do procedimento.

\begin{tabular}{cccccc}
\hline Etapas & Perfusão & Back-table & $\begin{array}{c}\text { Isquemia } \\
\text { fria }\end{array}$ & Anepática & $\begin{array}{c}\text { Cirurgia } \\
\text { Receptor }\end{array}$ \\
\hline Tempo (min) & 18,2 & 56,6 & 252,2 & 69 & 184,3 \\
\hline
\end{tabular}


O tempo consumido para realização das anastomoses da veia cava supra-hepática e da veia porta foram considerados satisfatórios, resultando em uma fase anepática relativamente curta, ideal para o procedimento. As outras anastomoses foram realizadas após a revascularização do fígado (tabela 2).

Tabela 2. Distribuição do tempo médio (em minutos) necessário para a realização das anastomoses.

\begin{tabular}{cccccc}
\hline Anastomose & VCSH & VP & VCIH & AH & VB \\
\hline Tempo $(\mathrm{min})$ & 16,07 & 16,14 & 19,8 & 17,7 & 6,22 \\
\hline
\end{tabular}

VCSH: Veia cava supra-hepática

VP: Veia porta

VCIH: Veia cava infra-hepática

$\boldsymbol{A H}$ : Artéria hepática

VB: Via biliar

Entre os parâmetros clínicos estudados em três diferentes fases no animal receptor, observamos alterações bem mais evidentes durante a fase de reperfusão do enxerto, caracterizada principalmente por queda na pressão arterial média (tabela 3).

Tabela 3. Distribuição do valor médio dos parâmetros clínicos observados no animal receptor.

\begin{tabular}{ccccc}
\hline Hemodinâmica & PAM & SO2 & FC & PVC \\
\hline Pré-anepática & 95,3 & 100 & 101,1 & 8,8 \\
Anepática & 85,4 & 95,2 & 119,8 & 9 \\
Reperfusão & 29,3 & 92,5 & 76 & 10 \\
\hline
\end{tabular}

PAM: Pressão arterial média

SO2: Saturação de oxigênio

FC: Freqüência cardíaca

PVC: Pressão venosa central

$\mathrm{Na}$ avaliação da gasometria destes animais receptores, identificamos alterações importantes na fase de reperfusão do enxerto, causadas ou por resposta cirúrgica ou por manipulação clínico-anestésica (tabela 4).

Tabela 4. Distribuição da média dos parâmetros gasométricos observados no animal receptor.

\begin{tabular}{cccccc}
\hline Gasometria & pH & pO2 & HCO3 & BE & pCO2 \\
\hline Pré-anepática & 7,37 & 169 & 25,9 & $-2,2$ & 49 \\
Anepática & 7,26 & 234 & 22,9 & $-4,1$ & 51 \\
Reperfusão & 7,28 & 194 & 17,0 & $-9,9$ & 44,7 \\
\hline
\end{tabular}

BE: Base excess

Os valores dos eletrólitos séricos no animal receptor demonstraram alterações, particularmente no sódio e cloro, tanto na fase anepática quanto na fase de reperfusão do enxerto (tabela 5).
Tabela 5. Valores médios dos eletrólitos séricos nas diferentes fases no animal receptor.

\begin{tabular}{llll}
\hline & Pre-anepática & Anepática & Reperfusão \\
\hline $\mathrm{Na} \mathrm{(mEq/l)}$ & $150( \pm) 6,96$ & $174,2( \pm) 15,82$ & $158,6( \pm) 16,43$ \\
$\mathbf{K}(\mathbf{m E q} / \mathbf{l})$ & $3,6( \pm) 0,39$ & $5,0( \pm) 0,91$ & $4,1( \pm) 0,64$ \\
$\mathrm{Cl} \mathrm{mEq} / \mathbf{l}$ & $100( \pm) 3,6$ & $153,5( \pm) 4,9$ & $132,3( \pm) 4,2$ \\
$\mathbf{C a ~ ( m m o l / L )}$ & $1,2( \pm) 0,11$ & $1,19( \pm) 0,09$ & $1,71( \pm) 0,18$ \\
\hline
\end{tabular}

\section{DISCUSSÃO}

O transplante hepático experimental, particularmente em suínos, tem sido realizado em diferentes centros como parte do preparo para realização do transplante clínico bem como para o desenvolvimento de modelos de pesquisa. O suíno apresenta considerável similaridade anatômica com o homem e comparável sensibilidade fisiológica e hemodinâmica, sendo também menos sujeito a restrições éticas. A principal diferença técnica tem sido a dificuldade de preservação da veia cava do receptor (técnica de Piggy-back). Este procedimento utilizando a técnica de Piggy-back em suínos não foi reproduzido no presente estudo. ${ }^{13,14,15,16,20,21}$

Realizamos o procedimento utilizando dois animais por transplante, sendo doador e receptor. O treinamento no animal doador proporciona aprendizado relacionado à captação de múltiplos órgãos. Outros autores realizam o procedimento com apenas um animal. $\mathrm{O}$ autotransplante de fígado pode apresentar algumas dificuldades técnicas, exigindo uma melhor dissecção dos elementos do pedículo. ${ }^{22,23}$

O by-pass veno-venoso passivo com o animal heparinizado durante a fase anepática, previne algumas alterações hemodinâmicas observadas devido ao clampeamento simultâneo do pedículo hepático e da veia cava infra-hepática. Este by-pass passivo evita a instabilidade hemodinâmica nos animais durante a fase anepática. Um outro procedimento que pode ser utilizado é o clampeamento da aorta supracelíaca durante a fase anepática. ${ }^{13,24}$

Uma padronização técnica para a realização de todo o procedimento deve ser bem definida, pois isto reflete na otimização do tempo e melhora dos resultados..$^{22,25,26,27}$ No presente estudo, por ser o procedimento do receptor realizado após a cirurgia do doador, observamos um aumento do tempo de isquemia fria. Entretanto, a fase anepática foi considerada aceitável, pois a perfusão ocorreu após a anastomose da veia porta. O tempo de 30 minutos tem sido considerado suficiente para a realização das duas anastomoses sem o by-pass veno-venoso, podendo ser facilmente tolerado pelo animal. . $0,11,22,25,27,28$

Apesar disto, preferimos utilizar o by-pass veno-venoso no presente estudo.

Clinicamente, os animais apresentam repercussões que dependem da fase do procedimento. Na fase anepática as alterações foram menos intensas que na fase de reperfusão no presente estudo. A pressão arterial média permaneceu estável durante toda a fase anepática, caindo de forma abrupta na fase de reperfusão do enxerto. O mesmo pode ser observado nos parâmetros gasométricos. O reaquecimento e reperfusão hepática parecem ser mais lesivos ao fígado, refletido por alterações importantes neste estudo. Nestas situações a acidose metabólica pode ser controlada pela administração de bicarbonato de sódio antes do desclampeamento vascular e correção das alterações dos eletrólitos séricos. ${ }^{13,14,24,26}$ Os valores laboratoriais registrados demonstram alterações nas fases anepática e de reperfusão do enxerto, particularmente o sódio e cloro. 
308 Orlando Jorge Martins Torres, Érica Sampaio Barbosa, Noelia Dias Carneiro Barros, Patrícia Brandão Pantoja, Elisabeth Teixeira Noguera Servin, Poliana Cristina Oliveira Moreira, Edson Dener Zandonadi Ferreira, Raquel Aranha Viegas, Cristiany de Almeida Barros, Santiago Cirilo Noguera Servin, José Aparecido Valadão, Tereza Rachel Vieira Gomes, Natalino Salgado Filho, Waston Gonçalves Ribeiro

\section{CONCLUSÃO}

Concluímos que o protocolo de transplante hepático experimental em suínos é capaz de otimizar a técnica cirúrgica identificando e corrigindo alterações clínicas e laboratoriais observadas bem com estimular pesquisas em transplante hepático.

\begin{abstract}
Objective: The aim of this study was to analyze the initials results of the experimental orthotopic liver transplantation in swine at the Maranhão Federal University. Material and Methods: From April 2004 to April 2005, it was performed 18 liver transplantations in thirtysix female swine, Landrace weighting between 32 and $38 \mathrm{Kg}$. All animals were divided in two groups: pairs of donors and recipients, which received whole liver grafts. The surgical procedure was divided in four parts: harvested, back-table, hepatectomy of the recipient and re-implantation. It was assessed the mean systemic arterial pressure, central venous pressure, heart rate, pO2, pCO2, $\mathrm{HCO} 3-$, base excess, $\mathrm{SO} 2, \mathrm{pH}, \mathrm{Na}+, \mathrm{K}+, \mathrm{Cl}-, \mathrm{Ca}++$. Also, it was recorded the perfusion, cold ischemia, surgery, back-table, anhepatic phase, and anastomosis. Results: It was found a long cold ischemia time, but the anastomosis of the supra-hepatic vena cava and portal vein were satisfactory, resulting in a short anhepatic phase time. Clinical and gasometric alterations were noted mainly during the reperfusion phase. It was also found alterations in the serum electrolytes, mainly in the sodium and cloride levels during the anhepatic phase and reperfusion. Conclusion: Experimental orthotopic liver transplantation in swine is able to improve the operative technique and to promote the development of research in liver transplant.
\end{abstract}

Key words: liver transplantation; experimental surgery; liver surgery; swine.

\section{REFERÊNCIAS}

1. Bambha K, Kim W R. Liver transplantation is effective, but is it cost-effective? Liver transplant 2003; 9:1308-311

2. Busuttil RW, Tanaka K. The utility of marginal donors in liver transplantation. Liver transplant 2003; 9; 651-63

3. Juan H, Innocenti FA- Liver transplantation in Latin America: current satus Transplant Proc 2004 36;1667-8.

4. Keeffe EB- Liver transplantation: current status and novel approaches to liver replacement. Gastroenterology 2001; 120:749-62.

5. Bacchella T, Machado MCC. The first clinical liver transplantation of Brazil revisited Transplant Proc 2004; 36: 929-30.

6. Starzl T, Marchioro TL, Von Kaulla KN, Hermann G, Brittain RS, Waddel WR Homotransplantation of the liver in humans. Surg Gynecol Obstet 1963; 117: 659-76.

7. Boillo O, Mechet I, Le DerfY, Bernard P, Figueiredo P, Berger F, et al. Portomesenteric disconnection for small-for-size grafts in liver transplantation: preclinical studies in pigs. Liver transplant 2003: 9: 42-6.

8. Laino GM, Anastasi A, Fabbri LP, Gandini E, Valanzano R, Fontanari P, et al Trapianto di fegato sperimentale nel maiale. Minerva Chir 1996; 51: 765-72.

9. Oike F, Uryuhara K, Otsuda M, Dehoux JP, Otte JB, Lerut J, et al. Simplified technique of orthotopic liver transplantation in pigs. Transplantation 2001; 71: 328-331.

10. Steinig, D Mentha G, Lecoultre C, Pittet JF, Jeanjacquot A, Huber O, et al. Experimental porcine orthotopic liver transplantation: a training protocol for transplantation in humans. Helv Chir Acta 1990; 57:177-86.

11. Tanaka K, Nishimura A, Ogata S, Yoshimine M, Ikoma A, Taira A- A simple method for liver transplantation in pigs. Transplantation 1994; 58: 1139-42.

12. Hojo, N, Ishibashi T, Yasuda T, Sakuma Y, Fujiwara T, Kawarasaki H, Nagai HPorcine model for surgical training of living related liver transplantation, Transplant Proc 2003; 35: 82-84

13. Nowak G, Ungerstedt J, Wernerman J, Ungerstedt U, Ericzon B. Metabolic changes in the liver graft monitored continuously with microdialysis during liver transplantation in a pig model. Liver transplant 2002: 8: 424-32.

14. Ott R, Schuppan N, Tannapfel A, Wittekind C, Erhardt W, Henke J, et al. Portal vein arterialisation as a technical option in liver transplantation: impact on function, regeneration, and morphology of the liver following hemihepatectomy in pigs. Liver Int 2003: 23: 54-62
15. Regueira FM, Espý A, Nwose P, Dýez-Caballero A, Baixauli' J, Rotellar F, et al. Comparison between two warm ischemic models in experimental liver transplantation in pigs. Transplant proc 2003; 35:1591-3.

16. Van AS AB, Lotz Z, Tyler M, Kahn D. Effect of early arterialization of the porcine liver allograft on reperfusion injury, hepatocellular injury, and endothelial cell dysfunction. Liver Transplant 2001; 7: 32-7.

17. Van AS AB, Lotz Z, Tyler M, Adams S, Ryffel B, Kahn D. Histological assessment after different methods of reperfusion following liver transplantation. S Afr J Surg 2002; $40: 95-8$

18. De Lange JJ, Hoitsma HF, Meijer S. Anaesthetic management in experimenta orthotopic liver transplantation in the pig. Eur Surg Res 1984; 16:360-65.

19. Eisele PH, Woodle ES, Hunter GC, Talken L, Ward RE. Anesthetic, preoperative and postoperative considerations for liver transplantation in swine. Lab Anim Sci 1986; 36: 402-05.

20. Filipponi F, Falcini F, Benassai C, Martini E. Orthotopic liver transplant in pigs: several variations of the surgical technic. G Chir 1989;10:374-8.

21. Miao X, Zhao H, Qi H. The model of piggyback orthotopic liver transplantation in pigs.HunanYi Ke Da XueXue Bao 1999; 24:77-9.

22. Gruttadauria,S Marino G, Catalano F, Sgroi AV, Di Mauro GL, Basile F. Porcine orthotopic liver autotransplantation: facilitated technique. J Invest Surg 2001; 14: 79-82.

23. Martinez-Ibanez V, Margarit-Creixell O, Boix-Ochoa A. A new experimental model of liver transplant in pigs. An Esp Pediatr 1987;26:107-10.

24. Lopez-Santamaria M, Migliazza L, Gamez M, Murcia J, Paz Cruz JA, Munoz J, et al Supraceliac aortic clamping during the anhepatic phase of experimental orthotopic liver transplantation.. J Pediatr Surg 1999; 34:1374-77.

25. Falcini F, Martini E, Marsili M, Benassai C, Fabbri LP, Tanini R. Veno-venous bypass in experimental liver transplantation: portal-jugular versus caval-portal-jugular. G Chir 1990;11:206-10.

26. Oldhafer KJ, Schuttler W, Hauss J, Spiegel HU, Pichlmayr R- Analysis of hepatic hemodynamics after orthotopic liver transplantation: an experimental study in pigs. Transplant Proc 1993; 25: 2598-9.

27. Taniguchi H, Takada K, Fukunaga K, Yuzawa M, Otsuka K, Todoroki F. Establishment of a swine model for auxiliary partial orthotopic liver transplantation. Transplant Proc 1998; 30: 3232-6.

28. Woodle ES, Hunter GC, Eisele P, Talken L, Ward RE. Orthotopic porcine liver transplantation: operative technique. J Surg Res 1985; 39 :483-8. 